
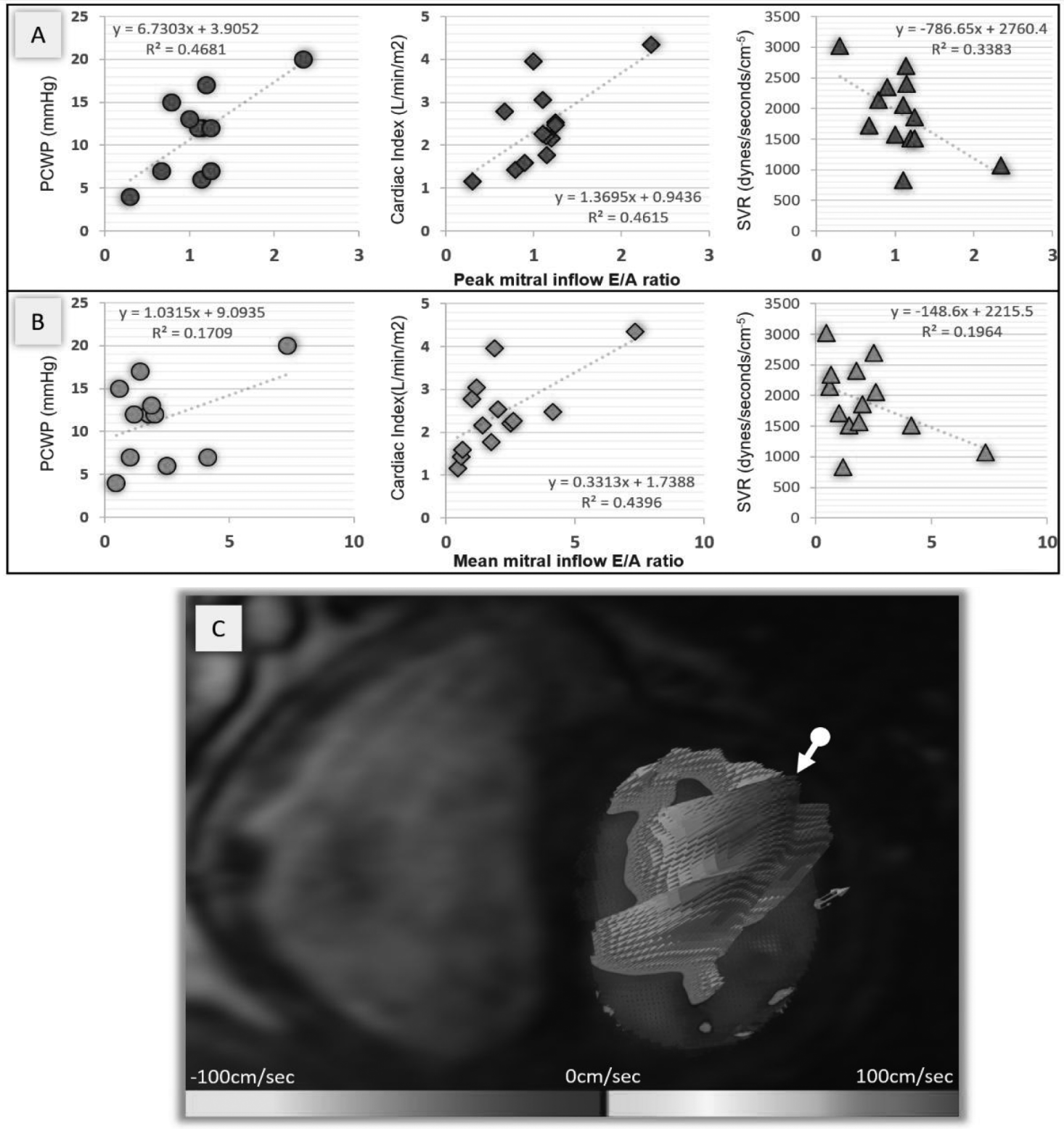

Abstract 25 Figure 1 Panel A: Scatter plots of invasive haemodynamic parameters to peak mitral inflow parameters. Panel B: Scatter plots of invasive haemodynamic parameters to mean mitral inflow parameters. Panel C: Phase contrast image overlay demonstrating signal intensity change of velocity through the mitral valve. The mean velocity is the average through the whole mitral valve and the peak is highlighted using the white arrow

reduced variation in through-plane peak velocity at different inflow levels versus the mean velocity. Future work is required to determine the effect of addressing the valvular throughplane motion, which may make the averaged mitral inflow assessment a better tool for LV hemodynamics.

\section{A NON-INVASIVE CMR ASSESSMENT FOR PREDICTING MEAN PULMONARY ARTERY PRESSURE IN PULMONARY HYPERTENSION}

${ }^{1}$ Benjamin Fidock, ${ }^{1}$ Nithin Balasubramanian, ${ }^{1}$ Natasha Barker, ${ }^{1}$ Alistair W Macdonald, ${ }^{1}$ David Capener, ${ }^{1}$ Christopher $S$ Johns, ${ }^{2}$ Kavitasagary Karunasaagarar, ${ }^{2}$ Graham Fent, ${ }^{2}$ Abdallah Al-Mohammad, 'Alexander Rothman, ${ }^{2}$ David G Kiely, 'Andrew Swift, 'James M Wild, 'Pankaj Garg. 'Department of Infection, Immunity and Cardiovascular Disease, University of Sheffield, Sheffield, UK; ${ }^{2}$ Sheffield Teaching Hospitals NHS Foundation Trust, Sheffield, UK

10.1136/heartjnl-2019-BSCMR.26
Background Pulmonary hypertension (PH) is defined by a resting mean pulmonary artery pressure (mPAP) $\geq 25 \mathrm{mmHg}$ by right heart catheterization. Systolic pulmonary artery pressures are used as surrogate non-invasive markers of $\mathrm{PH}$. A direct prediction tool of non-invasive mPAP would provide the capacity to diagnose disease through non-invasive means. It remains unclear if a multi-parametric cardiac magnetic resonance (CMR) integrated approach can provide an accurate measure of invasive mPAP.

Purpose This study sought to develop a novel CMR model using both established and newly derived CMR metrics for estimating mean pulmonary artery pressure.

Methods 18 patients were prospectively recruited at a large tertiary $\mathrm{PH}$ unit. All patients underwent right heart catheterisation (RHC) and CMR on the $1.5 \mathrm{~T}$ scanner (HDx scanner, GE Healthcare, Waukesha, Wisconsin, USA), using an 8-channel cardiac coil. CMR protocol included long and short axis cines and through-plane pulmonary artery phase contrast 

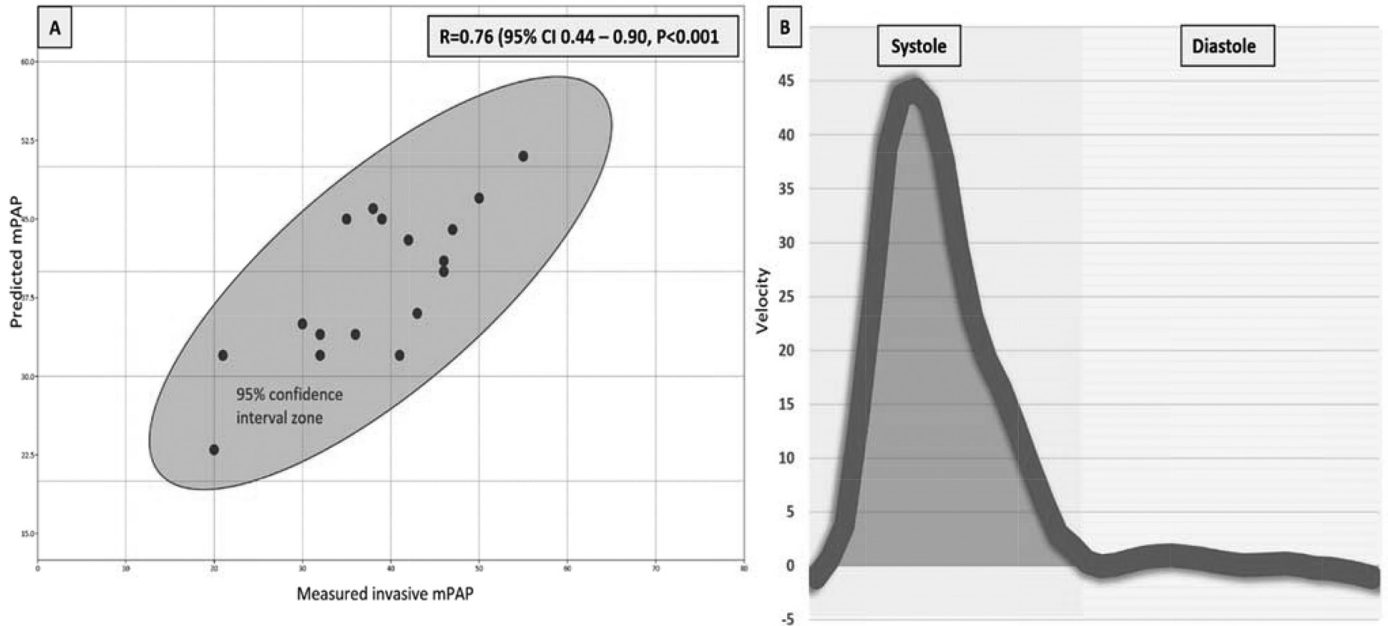

Abstract 26 Figure 1 Panel A: scatter plot of measured and predicted mean pulmonary artery pressure. Panel B: Main pulmonary artery (MPA) time-resolved, flow curves (the light blue area was used to compute mean pan-systolic area)

\begin{tabular}{llllll}
$\begin{array}{l}\text { Abstract 26 Table } 1 \\
\text { the study }\end{array}$ & \multicolumn{5}{l}{ Summary of CMR parameters evaluated in } \\
\hline \multicolumn{5}{l}{ Male (n=8) } & \multicolumn{4}{l}{ Female (n=10) } \\
\hline & Mean & SD & Mean & SD & P-value \\
\hline Age (Years) & 71.0 & 6.8 & 66.0 & 7.8 & 0.16 \\
Invasive mPAP (mmHg) & 36.4 & 9.5 & 41.9 & 8.9 & 0.23 \\
RA Area (cm ${ }^{2}$ ) & 23.7 & 6.1 & 28.1 & 11.8 & 0.31 \\
RVEDV (ml) & 165.3 & 51.1 & 202.3 & 72.6 & 0.22 \\
RVESV (ml) & 114.8 & 49.1 & 138.0 & 58.7 & 0.37 \\
RVSV (ml) & 50.7 & 25.5 & 64.4 & 22.7 & 0.25 \\
RVEF (\%) & 32.5 & 15.7 & 33.2 & 8.9 & 0.91 \\
PA SV (ml) & 50.0 & 24.7 & 53.6 & 16.8 & 0.72 \\
PA Mean Systolic & 15.0 & 6.9 & 14.3 & 3.4 & 0.79 \\
Velocity (cm/s) & & & & & \\
PA WSR & 39.5 & 22.1 & 36.6 & 10.2 & 0.74 \\
PA WSS & 138.1 & 77.3 & 127.9 & 35.2 & 0.73 \\
Predicted mPAP CMR & 37.5 & 7.4 & 40.5 & 6.8 & 0.40 \\
MODEL (mmHg) & & & & & \\
\hline
\end{tabular}

acquisition. The velocity encoded images were analysed for the following: mean pulmonary artery pan-systolic velocity (PASV), MPA stroke volume, MPA wall shear stress (WSS) and wall shear rate (WSR). The 4-chamber cine was used to measure end-diastolic right atrial area. Right ventricular volumes were analysed using standard methods. Stepwise multiple regression model of significantly associated parameters $(\mathrm{p}<0.05)$ was developed.

Results Mean age of the 18 patients was $68.78 \pm 7.46$ years (44\% males). The following CMR metrics demonstrated significant association to the measured mPAP: RA area $(r=0.65$ $\mathrm{p}=0.03)$; MPA mean pan-systolic velocity $(\mathrm{r}=-0.57 \mathrm{p}=0.01)$; RVEDV ( $\mathrm{r}=0.52 \mathrm{p}=0.03)$; RVEF $(\mathrm{r}=-0.40 \mathrm{p}=0.10)$; RVESV $(\mathrm{r}=0.58 \mathrm{p}=0.01)$. In stepwise multiple regression, only two parameters demonstrated independent association to mPAP RA Area and MPA mean pan-systolic velocity. The predicted mPAP demonstrated good correlation to the measured mPAP $(\mathrm{R}=0.76, \mathrm{p}<0.001)$.

Conclusion(s) Mean pulmonary artery systolic velocity and right atrial area are independently associated with mPAP.
Our novel CMR prediction model for mPAP, comprising of these two metrics, demonstrates high association to the measured mPAP by invasive haemodynamic study.

\section{MIXED VENOUS OXYGEN LEVELS IN PULMONARY HYPERTENSION IS ASSOCIATED WITH RIGHT HEART MULTI-PARAMETRIC ASSESSMENT}

Natasha Barker, Benjamin Fidock, Nithin Balasubramanian, Alistair W Macdonald, David Capener, Christopher S Johns, Kavitasagary Karunasaagarar, Graham Fent, Abdallah Al-Mohammad, Alexander Rothman, David G Kiely, James M Wild, Andrew Swift, Pankaj Garg. The University of Sheffield, Sheffield University teach hospitals NHS foundation trust

\subsection{6/heartjnl-2019-BSCMR.27}

Background Pulmonary hypertension (PH) is debilitating disease characterized by a progressive increase in pulmonary arterial pressure (PAP) that leads to right ventricular (RV) failure and death. Mixed venous oxygen saturation (SVO2) represents the oxygen saturation of blood returning to the lungs before reaching the alveolo-capillary units. SVO2 is strongly associated with clinical outcomes in $\mathrm{PH}$. The relationship of noninvasive CMR metrics to this prognostically relevant parameter in patients with $\mathrm{PH}$ are unknown.

Purpose This study sought to develop an early understanding of which CMR volumetric and flow parameters are most associated with SVO2.

Methods Eighteen $(n=18)$ patients were prospectively recruited at a large tertiary $\mathrm{PH}$ unit. The $\mathrm{SVO} 2$ was measured during right heart catheterisation. All patients had CMR on the 1.5 $\mathrm{T}$ scanner (HDx scanner, GE Healthcare, Waukesha, Wisconsin, USA), using an 8-channel cardiac coil. Subjects were scanned in the supine position with electrocardiogram (ECG) gating. CMR protocol included long and short axis cines and through-plane pulmonary artery phase contrast acquisition. The velocity encoded images were analysed for the following: mean pulmonary artery (MPA) systolic velocity, MPA stroke volume, MPA wall shear stress (WSS) and wall shear rate (WSR). The 4 chamber cine was used to measure end-diastolic right atrial (RA) area. RV volumes were analysed using standard methods. Stepwise multiple regression model of significantly associated parameters $(\mathrm{p}<0.05)$ was developed. 